\title{
Adaptive Maximums of Random Variables for Network Simulations
}

\author{
Rong Nan Chiou and Chia-Nian Shyi \\ Department of Computer Science and Information Engineering, Southern Taiwan University, \\ No. 1, Nan-Tai Street, Yung-Kang, Tainan 71005, Taiwan \\ Correspondence should be addressed to Rong Nan Chiou,rnchu@ms63.hinet.net
}

Received 22 October 2008; Accepted 19 January 2009

Recommended by Abdallah Shami

In order to enhance the precision of network simulations, the paper proposes an approach to adaptively decide the maximum of random variables that create the discrete probabilities to generate nodal traffic on simulated networks. In this paper, a statistical model is first suggested to manifest the bound of statistical errors. Then, according to the minimum probability that generates nodal traffic, a formula is proposed to decide the maximum. In the formula, a precision parameter is used to present the degree of simulative accuracy. Meanwhile, the maximum adaptively varies with the traffic distribution among nodes because the decision depends on the minimum probability generating nodal traffic. In order to verify the effect of the adaptive maximum on simulative precision, an optical network is introduced. After simulating the optical network, the theoretic average waiting time of nodes on the optical network is exploited to validate the exactness of the simulation. The proposed formula deciding the adaptive maximum can be generally exploited in the simulations of various networks. Based on the precision parameter $K$, a recursive procedure will be developed to automatically produce the adaptive maximum for network simulations in the future.

Copyright ( 2009 R. N. Chiou and C.-N. Shyi. This is an open access article distributed under the Creative Commons Attribution License, which permits unrestricted use, distribution, and reproduction in any medium, provided the original work is properly cited.

\section{Intoduction}

Simulations are an important technique for the design of systems, the estimation of performance, and the maintenance of systems $[1,2]$. It is widely used in various fields. For the simulation of complex systems, how to save computing time is an important topic. Moreover, it is also worthwhile to discuss how to reach acceptable simulative precision. In general, to promote simulative precision will lower simulative efficiency. Therefore, in order to enhance the simulative precision of complex systems, it is very important to take appropriate tradeoff between precision and efficiency.

For example, DQDB networks are systems with asynchronous transfer mode (ATM) and time-division multiple access (TDMA) [3]. Its medium access control (MAC) protocol is so complex that the performance analysis of the network is very difficult $[4,5]$. To make an exact analysis on the performance of the network is almost impossible [5]. Most papers estimate the performance of the network by simulations [6-20]. Thus, it only depends on the precision of simulations to exactly comprehend the behavior of the complex system. However, if simulative precision is overpromoted, simulative efficiency will be suppressed. Therefore, how to reach the acceptable simulative precision and meanwhile take good tradeoff between precision and efficiency is an important problem for simulating complex systems. It is worthwhile to explore, but there are few papers discussing the problem.

In simulations, random variables are used to create various distribution functions of probabilities. These probabilities are applied to direct the input amplitude of signals or noise as simulating communication systems $[1,2]$. For network simulations, probabilities are exploited to control the generation of nodal traffic. The probabilities assigned to represent nodal traffic are continuous. However, the probabilities created from random variables are discrete. Due to the inherent difference between the continuous and discrete probabilities, simulative errors will take place absolutely. 
In order to take precise simulations, this paper suggests a statistical model. Based on the model, it is obvious that simulative precision only depends on the maximum of random variables controlling the generation of network traffic. Based on the perception, a simple formula is proposed to decide the feasible lower bound of maximums of random variables. In the formula, the feasible lower bound is dependent on a precision parameter, denoted by $K$, and the minimum probability generating nodal traffic. The larger the precision parameter, the more the simulative precision. Then, a prime number that is slightly greater than the lower bound can be chosen as the maximum of random variables. The chosen maximum can adapt to the traffic distribution of simulated networks. So, the adaptive maximum cannot only result in acceptable simulative precision but also take desired efficiency. In practice, due to the approach deciding the adaptive maximum, simulative systems can make optimal tradeoff between reaching high precision and saving computing time.

So as to understand the effect of the adaptive maximum on simulative precision, an optical TDMA network is introduced. The MAC protocol of the optical TDMA network implements traffic control. The average waiting time of a node on the network is in inverse proportion to the traffic of the node [21]. Due to the quantitative analysis of the optical TDMA network, the root-mean-square (rms) difference between the simulative and theoretic average waiting times of nodes is calculated to validate the exactness of simulations.

In Section 2, the suggested statistical model exhibits the relationship between the statistical error and the maximum of random variables. The discussion for deciding the adaptive maximum of random variables will also be shown in this section. The MAC protocol performing traffic control and some working conditions assumed for simulating optical TDMA networks are presented in Section 3. Section 4 illustrates the effect of the adaptive maximum on the performance estimated by simulations. The validation of simulations will also be shown in this section. Section 5 includes conclusions.

\section{The Decision of Adaptive Maximums}

Before simulations, a set of continuous probabilities is assigned to predefine the distribution of nodal traffic. In simulations, a set of discrete probabilities, which corresponds to the set of continuous probabilities, controls the generation of nodal traffic. The difference between continuous and discrete probabilities is used to manifest the influence of the maximum of integral random variables on simulative precision. Then the minimum probability in the set of continuous probabilities is exploited to decide the adaptive maximum of integral random variables.

In simulated networks, every node has one queue. Queues consist of cell (packet) buffers. Queues provide firstin-first-out (FIFO) service. The first cell buffer in a queue is attached to the transmission system of the simulated network. When an available slot on the transmission system is passed through, the contents of the first cell buffer will be written into the available slot.
The number of cells temporarily storing in queues is dependent on the traffic generated by nodes. The heavier the nodal traffic, the longer the queuing delay. According to the complex MAC protocol of most networks, an available slot on transmission systems appears for some node randomly. Hence, the prediction of the queuing delay of a specified cell is difficult. In order to estimate performance, most simulations assume that networks are with heavy load $[4$, $6,11,21]$. This assumption will lengthen queues so that the theoretic analysis of the queuing delay of a specified cell will become more difficult. Therefore, how to enhance the accuracy of queuing delays is a key topic for network simulations. In order for taking precise simulations, a statistical model must be first introduced. Based on the model, the data resulted from simulations can be applied to calculate the nodal mean of queuing delays. The queuing delay is defined as the period for which a cell stays in queues.

For a node within networks, every cell generated by disassembling procedures is first stored in queues. Before the cell is transferred to transmission systems, it must be sequentially shifted into the first cell buffer of nodal queues. When the cell is within the first cell buffer, the MAC protocol will be exploited to decide the moment after that the node can write the cell out. In above operations, there are two moments relative to the queuing delay. The first moment is the instant that a cell enters queues. The second one is the flash that the cell is sent onto transmission systems. Let $I M_{i, j}$ and $O M_{i, j}$ in sequence denote the first and second moments of the $i$ th cell generated by the $j$ th node, where $i$ and $j$ are the ordinal number of cells and nodes, respectively. The queuing delay of the $i$ th cell generated by the $j$ th node is designated by $C_{i, j}$. Then $C_{i, j}$ can be represented as

$$
C_{i, j}=O M_{i, j}-I M_{i, j}, \quad 1 \leq i \leq \operatorname{Max}(R, j), 0 \leq j \leq N-1,
$$

where $\operatorname{Max}(R, j)$ is the ordinal number of the last cell generated by the $j$ th node, $R$ is the maximum of the integral random variable used to create the set of discrete probabilities, and $N$ is the number of nodes within networks.

Let $P_{I}$ denote the probability that the $I$ th node generates traffic. These probabilities, $P_{I}$ s, are chosen in accordance with interested simulative scenarios. The chosen probabilities are continuous, but their corresponding probabilities generated by an integral random variable are discrete. When a discrete probability corresponds to a continuous probability, both probabilities must be equal theoretically. But, in practice, the discrete probability is inherently different from its corresponding continuous probability. If the difference between them is large, statistical delays could not converge on the precise level that is acceptable.

Let $X$ represent an integral random variable that uniformly distributes over $[0, R]$. Its probability density function $f_{X}(x)$ can be presented by

$$
f_{X}(x)= \begin{cases}\frac{1}{R}, & 0 \leq x<R \\ 0, & \text { otherwise }\end{cases}
$$


The random variable $X$ is applied to create $P_{I} s$ for simulations. Let $\hat{P}_{I}$ denote the discrete probability corresponding to $P_{I}$. The difference between $\hat{P}_{I}$ and $P_{I}$ can be represented as

$$
\left|P_{I}-\hat{P}_{I}\right| \leq f_{X}(x)=\frac{1}{R}, \quad \text { where } \hat{P}_{I}=\frac{\operatorname{Max}(R, I)}{R} .
$$

From (3), if $R$ theoretically approaches infinite, the difference will become zero. Therefore, (3) can be rearranged as

$$
\lim _{R \rightarrow \infty} \hat{P}_{I}=P_{I}
$$

If a simulation is with a precise-probability set $\left\{\hat{P}_{I}=\right.$ $\left.P_{I}, I=0, \ldots, N-1\right\}$, the statistical delays of network simulations will also be precise. In a word, when $R$ theoretically approaches infinite, the statistical queuing delay will converge precisely.

After discussing the enhancement of statistical precision, statistics of the queuing delay are presented. According to (1), the $\left\{C_{i, j}\right\}$ is a set of positive random numbers. Let $\mu(j)$ denote the mean of $\left\{C_{i, j}\right\}$. Then $\mu(j)$ can be calculated by

$$
\mu(j)=\frac{1}{\operatorname{Max}(R, j)} \sum_{i=1}^{\operatorname{Max}(R, j)} C_{i, j} .
$$

The mean $\mu(j)$ represents the average queuing delay of the $j$ th node.

If $R$ is theoretically infinite, the computing time taken in simulations is also infinite. In practice, $R$ can be chosen in accordance with $P_{I} s$. If $P_{I} s$ is small, $R$ must be large enough to ensure statistical precision. In other words, $R$ must adaptively vary with $P_{I}$ s to promote simulative precision.

Let $P_{\text {min }}$ represent the minimum probability among $P_{I}$ s. $P_{\min }$ must be greater than the inverse of $R$ for minimum precise requirement in simulations, that is,

$$
P_{\min }>\frac{1}{R} \text {. }
$$

In order to guarantee that the precise degree is acceptable, $R$ must be chosen so that

$$
P_{\min } \geq \frac{K}{R},
$$

where $K>1$ is a positive real number.

Then, $R$, the adaptive maximum, can be represented by the equation

$$
R \geq \frac{K}{P_{\min }} .
$$

In (8), the value $K$ is a precision parameter. To enlarge $K$ will result in higher precision. On the other hand, if simulative procedures are with overlarge $K$, they will consume more computing time but promote little precision. Thus, the decision of the value $K$ is dependent on the acceptable degree of precision.

Because the recursive formula of the power-residue method is computationally very efficient $[1,22]$, it is widely adopted for generating random variables with uniform distribution. As the power-residue method is exploited to generate the uniform-distribution random sequence, the maximum of the integral random variable must be a prime number. Therefore, the lower bound of $R$, indicated by $R_{\mathrm{LB}}$, must be first taken with the following equation:

$$
R_{\mathrm{LB}}=\frac{K}{P_{\min }} .
$$

Then, a prime number that is slightly greater than $R_{\mathrm{LB}}$ can be assigned as the adaptive maximum $R$. The adaptive maximum can result in the optimal tradeoff between simulative precision and efficiency.

\section{Optical TDMA Networks}

In order for comprehending the effect of adaptive maximums of random variables on the precision of network simulations, an optical TDMA networks is introduced. Before depicting the structure of the network, the deduction for the average waiting time of nodes on the network (the waiting mean) is presented. The waiting time of a cell is the queuing delay that the cell waits in the first cell buffer of queues for an available slot on transmission systems. Based on the structure of the network, several working conditions are assumed for simulations. Due to these working conditions, an MAC protocol implementing traffic control is described.

For TDMA networks, a node must send requests to preserve empty slots when it is going to transmit messages. More requests preserve more slots. As the number of preserved slots of a node becomes large, the waiting mean of the node will be reduced. Therefore, if a node has more traffic, its waiting mean will be decreased. The relationship between the waiting mean and the traffic of the Ith node [21] can be presented as

$$
\mu(I)=\frac{\left[\begin{array}{c}
1-(1-T(I))^{S(1-T(I))+1} \\
(1+(S(1-T(I))+1) T(I))
\end{array}\right]}{T(I)},
$$

where $\mu(I)$ and $T(I)$ are the waiting mean and traffic of the $I$ th node, respectively, and $S$ is the slot rate of the optical TDMA networks.

Because optical TDMA networks are high-speed networks, the slot rate can approach infinite. Therefore, $\mu(I)$ can be rearranged as

$$
\begin{aligned}
\lim _{S \rightarrow \infty} \mu(I) & =\lim _{S \rightarrow \infty}\left\{\frac{\left[\begin{array}{c}
1-(1-T(I))^{S(1-T(I))+1} \\
(1+(S(1-T(I))+1) T(I))
\end{array}\right]}{T(I)}\right\} \\
& =\frac{1}{T(I)} .
\end{aligned}
$$

In (11), $\mu(I)$ is a function of $T(I)$. It exhibits that the waiting mean of a node on an optical TDMA network is in inverse proportion to the traffic of the node. The theoretic waiting mean will be exploited to validate simulations in Section 4. The structure of the optical TDMA network is shown in Figure 1. 


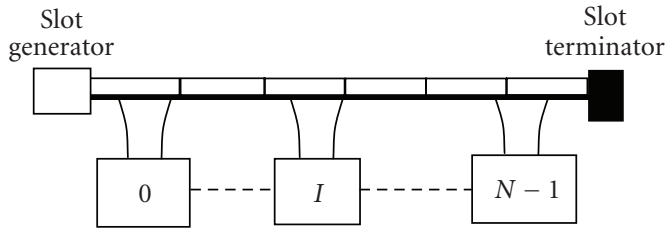

FIgURE 1: The structure of optical TDMA networks.
In Figure 1, the medium between the slot generator and the slot terminator is an optical fiber. The slot flow on optical fibers is sent by slot generators and sinks into slot terminators. The number of nodes within the network is $N$. Nodes are numbered from 0 to $(N-1)$ in sequent order. The ordinal number of every node also relates to the nodal position in the topology. The period that the slot generator just completely sends a slot onto optical fibers is called a slot time. A slot length is the distance that a slot spreads on the optical fiber. Other working conditions concerning the space between adjacent nodes, the length of messages, and the traffic distribution among nodes are described as follows.

For all interested simulative scenarios, the space between adjacent nodes is one slot length. Messages are similar in length. Every message can be contained in the payload of a slot.

The traffic distribution among nodes affects the operation of traffic control in the MAC protocol. For the benefit of easily performing traffic control, a basic traffic denoted by $T_{B}$ is introduced. The amount of $T_{B}$ is dependent on the defined distribution of traffic. In a scenario, the traffic of some node can be several times the amount of $T_{B}$. In order to obviously present the influence of the adaptive maximum on simulative precision, it is assumed that traffic is uniform distribution among nodes in every simulative scenario. Hence, the traffic of every node in a simulative scenario is equal to one $T_{B}$. Because the optical fiber is a one-way bus, and all messages are not transmitted out of the network, the $(N-1)$ th node does not generate any traffic. Let $T_{N}$ denote the network traffic. Then $T_{N}$ can be presented by

$$
T_{N}=\sum_{I=0}^{N-2} T(I)=(N-1) T_{B}
$$

Therefore, the $T_{B}$ in simulations can be shown as

$$
T_{B}=\frac{T_{N}}{(N-1)} .
$$

Based on the introduction of $T_{B}$, the approach of traffic control can be described as follows.

In this paper, slot frames are used to implement traffic control. The slot flow on optical fibers is partitioned into frames. There are $1 / T_{B}$ slots in a frame. When a frame is passed to the Ith node, the node can only write one message into an empty slot within the frame when its queue is not empty. After the operation, the node must immediately stop writing messages out regardless of whether its queue is empty or not. After the moment, the node waits for the arrival of the next frame to restart the controlling process.
TABLE 1: Relative parameters in two scenarios.

\begin{tabular}{cccccc}
\hline$T_{N}$ & $N$ & $P_{\min }=T_{B}$ & $K$ & $R_{\mathrm{LB}}$ & $R$ \\
\hline & & & 300 & 46800 & 46807 \\
& 40 & 0.006411 & 3000 & 468000 & 468001 \\
& & & 30000 & 4680000 & 4680001 \\
0.25 & & & 300 & 58800 & 58831 \\
& \multirow{2}{*}{50} & \multirow{2}{*}{0.005102} & 3000 & 588000 & 588011 \\
& & & 30000 & 5880000 & 5880023 \\
\hline
\end{tabular}

\section{Simulations}

The simulation is applied to present the influence of the adaptive maximum of a random variable on simulative precision. The waiting mean of optical TDMA nodes manifests the precise degree caused by different adaptive maximums. The simulative efficiency is dependent on the size of the adaptive maximum. The larger the maximum of the random variable, the lower the efficiency of the simulative system. On the other hand, the theoretic waiting mean calculated by (11) will be used to validate simulative data. The rms difference between the simulative and theoretic data, denoted by $D_{\mathrm{rms}}$, is defined as

$$
D_{\mathrm{rms}}=\left(\frac{1}{N-1} \sum_{I=0}^{N-2}\left(\mu_{s}(I)-\mu(I)\right)^{2}\right)^{1 / 2},
$$

where $\mu_{s}(I)$ and $\mu(I)$ are the simulative and theoretic waiting means of the Ith node, respectively.

Two parameters must be chosen before simulations. The parameters are the number of nodes $N$ and the network traffic $T_{N}$. The chosen $N s$ and $T_{N} s$ are based on interested scenarios. After the choice of $N s$ and $T_{N} s,(13)$ can be exploited to calculate the basic traffic $T_{B}$. Because messages are one slot in length, the basic traffic $T_{B}$ can be regarded as the minimum probability $P_{\min }$. In order for clearly distinguishing between precise degrees corresponding to different adaptive maximums, small $P_{\min }$ is necessary. So, the chosen $T_{N}$ in all interested scenarios is equal to 0.25 .

In simulations, two scenarios are interested. In order to certainly manifest the effect of adaptive maximums, the Ns in two scenarios are 40 and 50, respectively. On account of sufficiently exhibiting the influence of precision parameters on simulative precise degrees, three $K$ s are assigned in every scenario. The three $K s$ in every scenario are 300,3000 , and 30000 , respectively. Then, (9) is used to calculate the corresponding $R_{\mathrm{LB}}$ of every $K$ parameter. In accordance with the power-residue method, the adaptive maximum $R$, which is a prime number and slightly greater than its corresponding $R_{\mathrm{LB}}$, can be finally found. According to the description above, those relative parameters derived from the $T_{N}, N s$, and $K s$ are listed in Table 1.

For traffic control, the number of slots in a frame, which is equal to $1 / T_{B}$, must vary with scenarios. Due to (13), the number of slots in a frame, denoted by $N_{\mathrm{SF}}$, can be presented as

$$
N_{\mathrm{SF}}=\frac{(N-1)}{T_{N}} .
$$




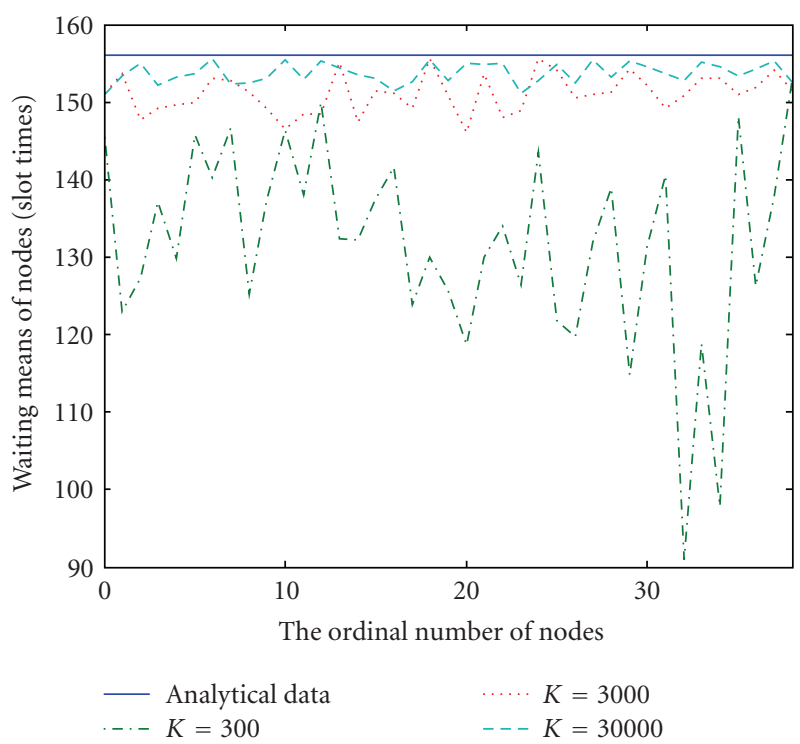

FIGURE 2: Waiting means of 40-node optical TDMA networks.

TABle 2: $D_{\text {rms }}$ s corresponding to Ks and Ns.

\begin{tabular}{cccc}
\hline$N$ & \multicolumn{3}{c}{ K } \\
& 300 & 3000 & 30000 \\
\hline 40 & 27.40495 & 5.489945 & 2.63133 \\
50 & 40.48827 & 8.349287 & 3.369718 \\
\hline
\end{tabular}

Hence, the $N_{\mathrm{SF}}$ of the scenario with 40 nodes is 156 and that of the scenario with 50 nodes is 196. Because uniform traffic distribution is assumed, the traffic of every node is equal to the $T_{B}$ in each scenario. Consequently, the theoretic waiting mean calculated by $(11)$ is the same as the $N_{\mathrm{SF}}$ in each scenario.

In the following figures that show simulative results, the horizontal axis is the ordinal number of nodes. Because the ordinal number of nodes is discrete, all curves in figures consist of piecewise lines. The waiting mean of nodes on the vertical axis is expressed in slot times.

After simulations, (5) is used to calculate the simulative waiting mean of nodes. Figures 2 and 3 show the variation of waiting means corresponding to two scenarios, respectively. In every figure, the horizontal solid line represents the theoretic waiting mean. Other three curves are relative to simulative data corresponding to three precision parameters. In two figures, it is obviously exhibited that curves will become smoother when the precision parameter $K$ is enlarged.

Based on the theoretic waiting mean, $D_{\mathrm{rms}} \mathrm{s}$ calculated by (14) is used to validate the simulations. Table 2 presents these $D_{\text {rms }}$ s. Every $D_{\text {rms }}$ represents the rms difference between the horizontal solid curve and a simulative curve in each figure. Observing Table 2, larger precision parameter $K$ and smaller $N$ will result in smaller $D_{\text {rms }}$. Therefore, a simulative curve will approach the horizontal solid line if precision parameter $K$ is consecutively enlarged. However, the decrease

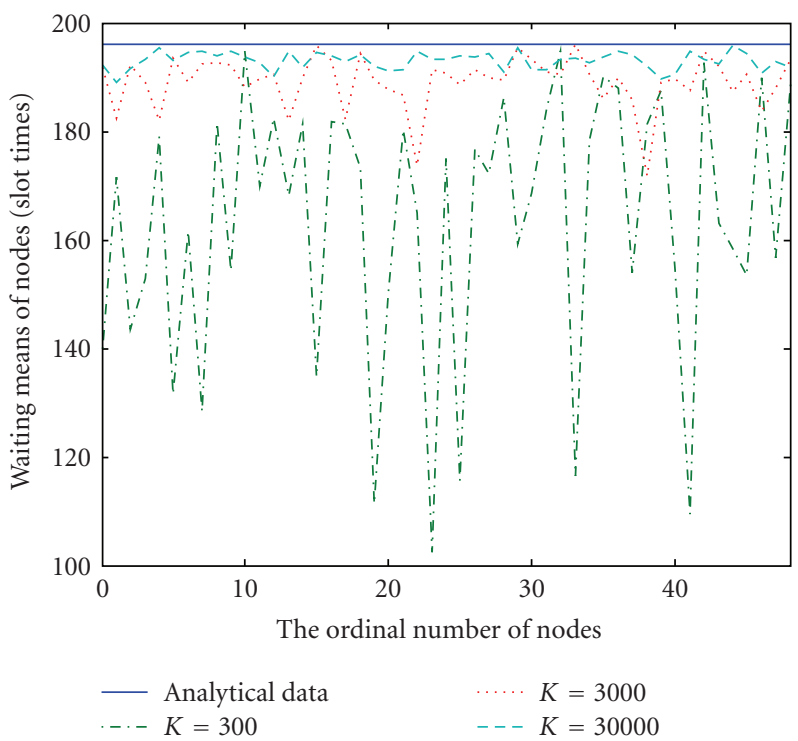

FIGURE 3: Waiting means of 50-node optical TDMA networks.

of $D_{\mathrm{rms}} \mathrm{s}$ does not linearly correspond to the increase of $K$ parameters. Consequently, simulative procedures with overlarge $K$ will consume more computing time but promote little precision. To adjust $K$ to a proper value can take an adaptive maximum to result in an acceptable precise level with relative high efficiency. In a word, network simulations with appropriate adaptive maximums can take optimal tradeoff between simulative precision and efficiency.

\section{Conclusions}

The paper discusses tradeoff between simulative precision and efficiency. Based on the statistical model of queuing delays, the difference between continuous and discrete probabilities is used to manifest the effect of the maximum of random variables on the statistical error. Then a simple method with precision parameter $K$ is proposed to decide the maximum of random variables. The maximum adapts to the minimum probability among $P_{I}$ s. The adaptive maximum must be enlarged as the minimum probability of $P_{I}$ s becomes smaller.

In order to manifest the effect of the adaptive maximum on the simulative precision, an optical TDMA network whose MAC protocol performs traffic control is simulated. The average waiting time of some optical TDMA node is in inverse proportion to the traffic of the node. The theoretic average waiting time is exploited to calculate $D_{\mathrm{rms}} s$ to validate the exactness of simulations. Simulative results exhibit that the adaptive maximum can take optimal tradeoff between simulative precision and efficiency.

In network simulations, the adaptive maximum not only results in the acceptable degree of precision but also suitably saves computing time. Based on the precision parameter $K$, a recursive procedure will be developed to automatically generate the adaptive maximum in future. 


\section{References}

[1] M. C. Jeruchim, P. B. Balaban, and K. S. Shanmugan, Simulation of Communication Systems: Modeling, Methodology, and Techniques, Kluwer Academic Publishers, New York, NY, USA, 2000.

[2] C. B. Rorabaugh, Simulating Wireless Communication Systems, Pearson Education, Upper Saddle River, NJ, USA, 2004.

[3] IEEE Std 802.6, "Distributed Queue Dual Bus (DQDB) Subnetwork of a Metropolitan Area Network (MAN)," December 1990.

[4] C. Y. R. Chen, G. A. Makhoul, and D. S. Meliksetian, "A queueing analysis of the performance of DQDB," IEEE/ACM Transactions on Networking, vol. 3, no. 6, pp. 872-881, 1995.

[5] B. Mukherjee and S. Banerjee, "Alternative strategies for improving the fairness in an analytical model of DQDB networks," in Proceedings of the 10th Annual Joint Conference of the IEEE Computer and Communications Societies (INFOCOM '91), vol. 2, pp. 879-888, Bal Harbour, Fla, USA, April 1991.

[6] R. N. Chiou and J. K. Guo, "The effect of bursty lengths on DQDB networks," in Proceedings of the 1st International Conference on Complex, Intelligent and Software Intensive Systems (CISIS '07), pp. 85-91, Vienna, Austria, April 2007.

[7] R. N. Chiou and J. K. Guo, "The characteristics of queuing delays on DQDB networks," in Proceedings of the International Symposium of Photonic Devices and System Application, pp. 2526, Taipei, Taiwan, April 2006.

[8] S. M. Banik, S. Radhakrishnan, T. Zheng, and C. N. Sekharan, "Distributed floor control protocols for computer collaborative applications on overlay networks," in Proceedings of the International Conference on Collaborative Computing: Networking, Applications and Worksharing, pp. 1-10, San Jose, Calif, USA, December 2005.

[9] N. A. EL-Fishawy and L. M. Zawra, "Performance evaluation of DQDB+/- metropolitan area networks supporting isochronous and nonisochronous traffic," in Proceedings of the 20th National Radio Science Conference (NRSC '03), pp. 1-8, Cairo, Egypt, March 2003.

[10] N. Funabiki, A. Sugano, and T. Higashino, "A proposal of a minimal-state processing search algorithm for isochronous channel reuse problems in DQDB networks," in Proceedings of the 17th IEEE International Conference on Advanced Information Networking and Applications (AINA '03), pp. 229-232, Xian, China, March 2003.

[11] T.-J. Kim and D.-H. Cho, "Bandwidth tuning for fairness of DQDB in client-server traffic environments," IEEE Communications Letters, vol. 4, no. 6, pp. 208-210, 2000.

[12] R. G. de Silva and W. J. Dewar, "Congestion control in DQDB/ATM interworking using PWM," in Proceedings of the IEEE Global Telecommunications Conference (GLOBECOM '98), vol. 3, pp. 1527-1532, Sydney, Australia, November 1998.

[13] G. B. Brewster and M. K. Vernon, "The fairness of DQDB networks with slot reuse," in Proceedings of the 14h Annual Joint Conference of the IEEE Computer and Communication Societies (INFOCOM '95), vol. 3, pp. 1154-1163, Boston, Mass, USA, April 1995.

[14] W. M. Moh, Y.-J. Chien, I. Zhang, and T. Moh, "Delay performance evaluation of high speed protocols for multimedia communications," in Proceedings of the 4th International Conference on Computer Communications and Networks (ICCCN '95), pp. 352-355, Las Vegas, Nev, USA, September 1995.
[15] L. N. Kumar and A. D. Bovopoulos, "An access protection solution for heavy load unfairness in DQDB," in Proceedings of the 11th Annual Conference of the IEEE Computer and Communications Societies (INFOCOM '92), vol. 1, pp. 190199, Florence, Italy, May 1992.

[16] S. Y. Cheung, "Controlled request DQDB: achieving fairness and maximum throughput in the DQDB network," in Proceedings of the 11th Annual Conference of the IEEE Computer and Communications Societies (INFOCOM '92), vol. 1, pp. 180189, Florence, Italy, May 1992.

[17] M. Sumita, P. C. Fetterolf, and A. D. Little, "Effect of bandwidth balancing mechanism on fairness and performance of DQDB MANs," in Proceedings of the 11th Annual Conference of the IEEE Computer and Communications Societies (INFOCOM '92), vol. 3, pp. 1096-2005, Florence, Italy, May 1992.

[18] K. H. Liang and Y. C. Chen, "An approach for fairness improvement in DQDB networks," in Proceedings of the $3 \mathrm{rd}$ Workshop on Future Trends of Distributed Computing Systems, pp. 165-171, Taipei, Taiwan, April 1992.

[19] E. L. Hahne, A. K. Choudhury, and N. F. Maxemchuk, "DQDB networks with and without bandwidth balancing," IEEE Transactions on Communications, vol. 40, no. 7, pp. 11921204, 1992.

[20] J. Filipiak, "Access protection for fairness in a distributed queue dual bus metropolitan area network," in Proceedings of the IEEE International Conference on Communications (ICC '89), vol. 2, pp. 635-639, Boston, Mass, USA, June 1989.

[21] R. N. Chiou and C. N. Shyi, "The performance of optical networks with TDMA," in Proceedings of the 2nd International Multi-Conference on Engineering and Technological Innovation (IMETI '09), Orlando, Fla, USA, July 2009.

[22] R. Y. Rubenstein, Simulation and the Monte-Carlo Method, John Wiley \& Sons, New York, NY, USA, 1981. 

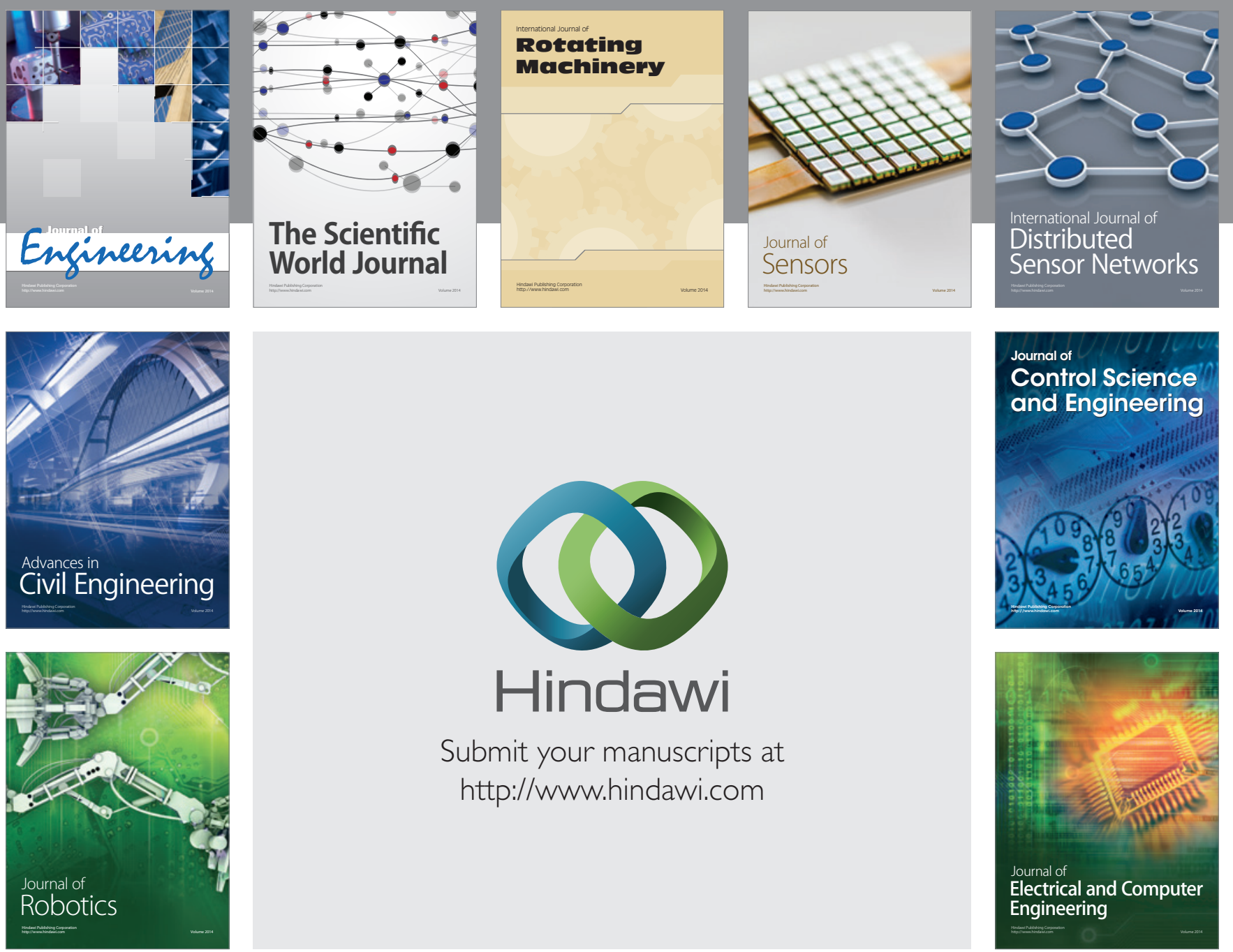

Submit your manuscripts at

http://www.hindawi.com
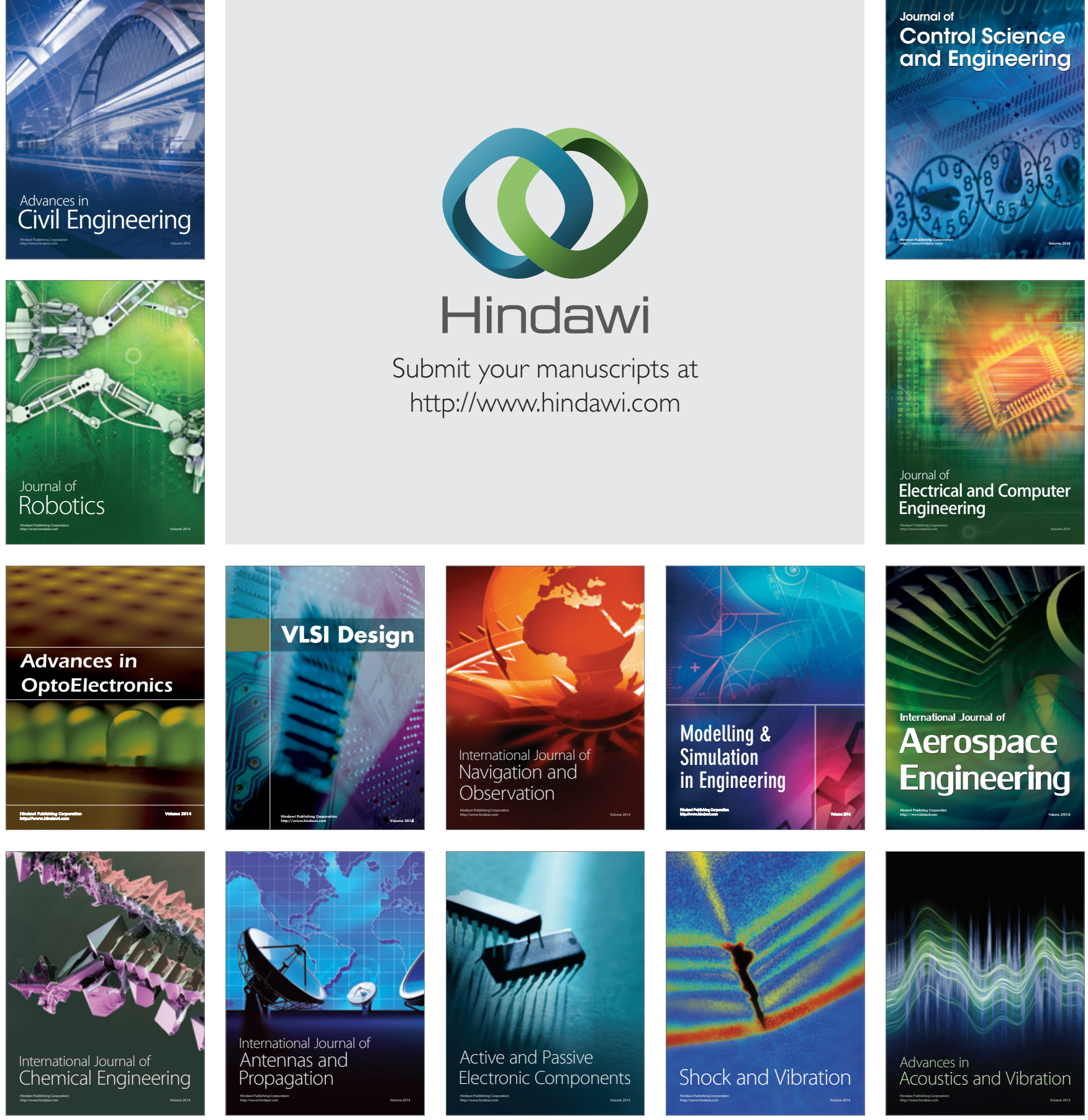